\title{
Interfacial effects in nano-silica/polypropylene composites fabricated by in-situ chemical blowing
}

\author{
L. F. Cai ${ }^{1,2,3}$, Y. L. Mai ${ }^{3}$, M. Z. Rong', W. H. Ruan ${ }^{2}$, M. Q. Zhang2* \\ ${ }^{1}$ Key Laboratory for Polymeric Composite and Functional Materials of Ministry of Education, OFCM Institute, School of \\ Chemistry and Chemical Engineering, Zhongshan University, Guangzhou 510275, P. R. China \\ ${ }^{2}$ Materials Science Institute, Zhongshan University, Guangzhou 510275, P. R. China \\ ${ }^{3}$ Guangdong Public Laboratory of Chemical Engineering, Guangzhou 510665, P. R. China
}

Received 23 October 2006; accepted in revised form 15 November 2006

\begin{abstract}
By mixing macromolecular blowing agent grafted nano- $\mathrm{SiO}_{2}$ with polypropylene (PP) melt, the nanoparticle agglomerates can be pulled apart due to the in-situ bubble-stretching resulting from gasification of the side foaming groups on the grafted polymer. The present work evaluated the interfacial effect in the PP based nanocomposites prepared using the aforesaid technique through introducing rubbery components to the backbone of the grafted polymer chains. The results indicated that deagglomeration of the nanoparticles was not bound to yield the highest properties of the composites. The positive effect of the nanoparticles was brought into full play because of the joint contributions of particles dispersion status and interfacial interaction. An interlayer with proper flexibility ensured an overall enhancement of mechanical properties, especially impact strength, of the nanocomposites.
\end{abstract}

Keywords: nanomaterials, processing technologies, nanoparticles, nanocomposites, interfacial effect

\section{Introduction}

Polymer/inorganic nanocomposites prepared via melt compounding have become a popular topic in material science because of their markedly improved properties and relatively facile preparation procedures $[1,2]$. Enhanced characteristics in such composites have so far been reported as a synergistic contribution resulting from the effective combination of nanoparticles and polymer matrix. However, whether nanoparticles are able to bring positive effects into play depends on their dispersibility in the matrix. Because of the high surface energy, these particles have a great inclination for agglomeration, especially in polymer melts that are characterized by high viscosity. Therefore, preparation of nanocomposites with uniformly distributed nanoparticles is a challenging task [3].
Recently, a novel approach for preparing nanoparticles/polymer composites was developed in the authors' lab based on in-situ bubble-stretching model [4]. That is, macromolecular blowing agent, poly(p-vinylphenylsulfonylhydrazide), was firstly grafted onto nano-silica [5]. The foamable side groups were then gasified during melt compounding with polypropylene (PP). The rapid inflation of the surrounding matrix is able to effectively pull apart the agglomerated nanoparticles, while the remaining backbone of the grafted polymer helped to strengthen the filler/matrix interaction through chain entanglement and interdiffusion at the interface. As a result, mechanical properties of the PP composites were significantly improved. Owing to the trace dosage of the attached foaming groups, the amount of the purposely produced polymer bubbles were kept at rather low level, so that

*Corresponding author, e-mail: ceszmq@mail.sysu.edu.cn

(c) BME-PT and GTE 
defoaming of the compounds was not necessary prior to injection molding. It implies that this technique could lead to deagglomeration of the nanoparticles when nanocomposites are being manufactured, without side effect that might deteriorate performance.

For polymer-based nanocomposites, an appropriate surface treatment of inorganic nanoparticles should not only improve dispersion of the fillers, but also bring about notable influence on the interfacial characteristics, and subsequently enhance the mechanical properties of the ultimate composites [6]. Considering that graft treatment of nanoparticles leads to specific interfacial structures that can be tailored by changing graft monomers and graft conditions [7], the authors of the present work planned to introduce polymer chain units with relatively higher molecular mobility (i.e., poly(butyl acrylate)) into the aforesaid grafted polymeric foaming agent poly(p-vinylphenylsulfonylhydrazide) through copolymerization. It is hoped that the stiffness of the interlayer in the nanocomposites originally constructed by the grafted poly(p-vinylphenylsulfonylhydrazide) containing rigid phenyl groups can be somewhat balanced.

In accordance with this idea, poly(p-vinylphenylsulfonylhydrazide-co-butyl acrylate) grafted nanosilica was synthesized. Afterwards, the treated nanoparticles were melt compounded with PP. With the help of in-situ bubble-stretching effect and the flexible interphase, agglomerated silica nanoparticles should be disconnected from each other and adhered to the matrix polymer via the grafted copolymer chains (Figure 1). In this paper, the feasibility of this technical route was analyzed by characterizing the grafted nano-silica and their influence on the structure and properties of PP based composites.

\section{Experimental}

\subsection{Materials}

Silica (Aerosil 200) was supplied by Degussa Co., Germany with an average diameter of $12 \mathrm{~nm}$ and a specific surface area of $200 \mathrm{~m}^{2} / \mathrm{g}$. The particles were dried at $120^{\circ} \mathrm{C}$ under vacuum for $24 \mathrm{~h}$ to eliminate the physically absorbed and weakly chemically absorbed species. Isotactic PP homopolymer (trade name: HZ730L) with melt flow index (MI) of $5.52 \mathrm{~g} / 10 \mathrm{~min}$ (ASTM D569-90), provided by Samsung ATOFINA Co. Ltd., Korea, was used as the matrix polymer of the nano-silica filled composites in this work.

Silane coupling agent ( $\gamma$-methacryloxypropyl trimethoxy silane, trade name: KH570) was provided by Liao Ning Gaizhou Chemical Industry Co. Ltd., China. 4-styrenesulfonic acid sodium salt hydrate was obtained from Aldrich Chemical Company, Inc., USA. Butyl acrylate (BA) monomer was distilled under low pressure prior to the graft polymerization. N, N-dimethylformamide (DMF) was purified to obtain anhydrous solvent. 2, 2 '-azobisisobutyronitrile (AIBN) was recrystallized from ethanol before use.

\subsection{Synthesis of grafted nano-SiO}

The procedure of grafting of poly(p-vinylphenylsulfonylhydrazide-co-butyl acrylate) onto nanosilica was similar to that of grafting poly(p-vinyl-

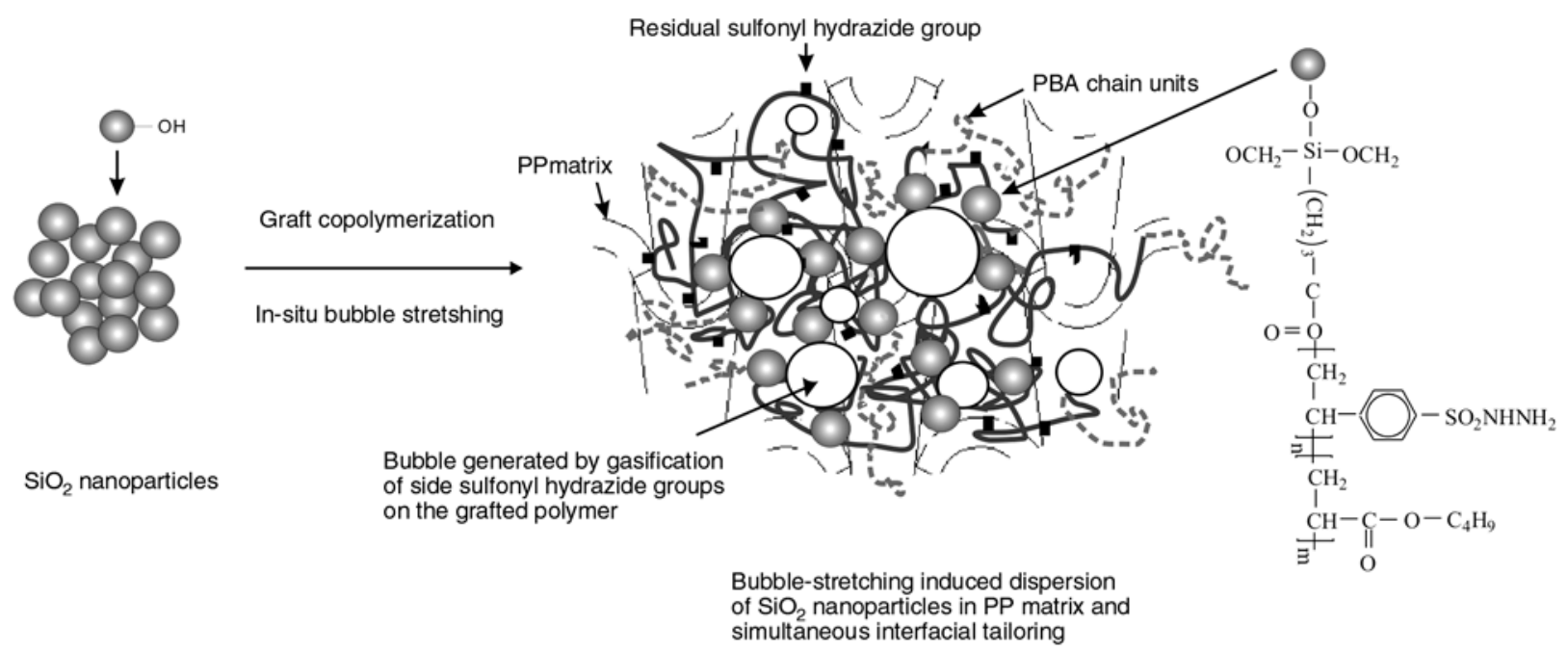

Figure 1. Schematic drawing of the proposed route for making nano- $\mathrm{SiO}_{2} / \mathrm{PP}$ composites 
phenylsulfonylhydrazide) onto nano-silica, which has been described elsewhere [5], despite the fact that butyl acrylate was charged in one batch after pvinylphenylsulfonylhydrazide had been polymerized for a period of time. A NETZSCH TG 209C thermogravimetric analyzer was used to determine percentage grafting of the grafted nanosilica, and a JXA-8800R electron probe micro-analyzer was employed to investigate the composition the grafted copolymer. Accordingly, poly(p-vinylphenylsulfonylhydrazide-co-butyl acrylate) grafted nano-silica (with a percent grafting of $13.58 \%$, and poly(p-vinylphenylsulfonylhydrazide/poly(butyl acrylate $)=1.00 / 2.63$ (weight ratio) $)$ was used for the subsequent characterization and composites preparation.

\subsection{Composites preparation}

To prepare PP composites, ungrafted or grafted nano-silica was compounded with PP in the mixer of a Haake Rheocord 300p torque rheometer at $180^{\circ} \mathrm{C}$ for $10 \mathrm{~min}$. Rotation speed of the mixing rotors was set at $60 \mathrm{rpm}$. Then, the mixture was injection molded into nanocomposites standard bars for mechanical tests with a Y-350 vertical injection molding machine. The dimensions of deformable region of tensile dumbbell specimens are $25.0 \times 3.2 \times 2.0 \mathrm{~mm}^{3}$, while those of the rectangular bars for testing impact strength are $80 \times 10 \times 4 \mathrm{~mm}^{3}$. The bars were further notched in the middle using a CSI cutter to produce ' $\mathrm{V}$ ' type notches with a depth of $2 \mathrm{~mm}$.

\subsection{Characterization}

Fourier-transform infrared (FTIR) spectroscopy was employed to investigate the variation in surface chemical structure of the nanoparticles. The spectra were recorded in $\mathrm{KBr}$ pellets on a Nicolet 5DX FTIR spectrometer. Blank scanning was performed before measurements to eliminate the influence of water vapor and $\mathrm{CO}_{2}$ in air.

Thermal degradation measurements were performed on the aforesaid thermogravimetric analyzer from 30 to $800^{\circ} \mathrm{C}$ at a heating rate of $10^{\circ} \mathrm{C} / \mathrm{min}$ under the flow of anhydrous air.

To assess dispersion of the nanoparticles in PP matrix, ultrathin sections of the compounds obtained from the melt mixing were examined by a
FEI TECNAI 12 transmission electron microscope (TEM) at an accelerating voltage of $100 \mathrm{kV}$.

Tensile properties of the nanocomposites were determined with a Hounsfield 10K-S universal tester at a crosshead speed of $50 \mathrm{~mm} / \mathrm{min}$ according to ASTM D638-98. Notched Charpy impact strength was measured by an Atlas advanced pendulum impact device in accordance with ISO 179-2. All of the specimens were stored at $30^{\circ} \mathrm{C}$ and $50 \%$ relative humidity for $48 \mathrm{~h}$ in advance.

\section{Results and discussion}

Prior to study of the nanocomposites, surface feature of the grafted nano-silica should be known. It is shown in Figure 2 that in comparison with the spectrum of nano-silica as-received, the additional characteristic adsorptions of carbonyl groups $\left(\sim 1730 \mathrm{~cm}^{-1}\right)$, phenyl $\left(\sim 576 \mathrm{~cm}^{-1}\right.$ and $1458 \mathrm{~cm}^{-1}$, respectively) and $\mathrm{C}-\mathrm{H}$ stretching modes (multiple peaks at 2820 2962 $\mathrm{cm}^{-1}$ ) appear on the spectrum of grafted nano-silica. Evidently, poly(p-vinylphenylsulfonylhydrazide-co-butyl acrylate) has been covalently bonded to the nanoparticles as expected.

Since the modified nanoparticles are intended to bring the macromolecular foaming agent into PP, pyrolytic behaviors of the related substances were measured (Figure 3). The visible differences among the grafted nano-silica, untreated nano-silica and silane treated silica should be indicative of the role of the grafted polymer. There is an obvious weight loss at around $138^{\circ} \mathrm{C}$ on the thermogram of the grafted nanoparticles, which is similar to the

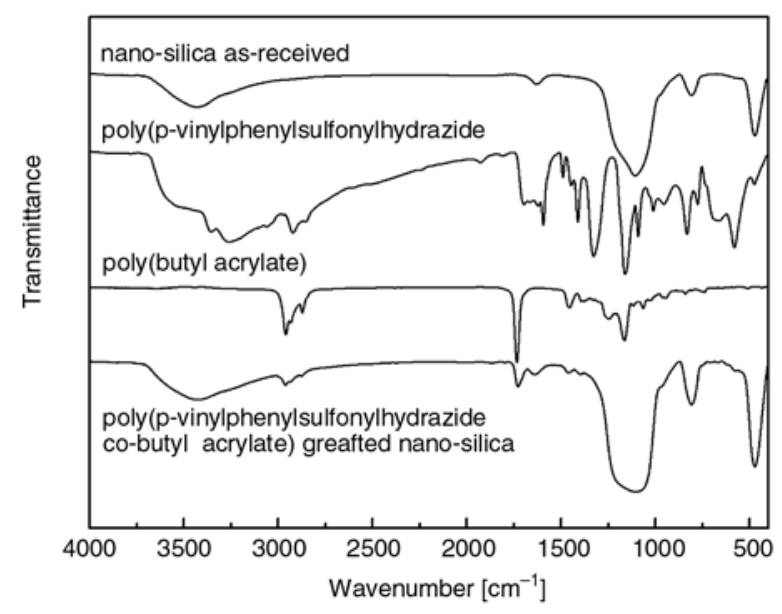

Figure 2. FTIR spectra of the related materials. For the grafted nano-silica, the ungrafted polymer had been removed by solvent extraction before the FTIR measurement 


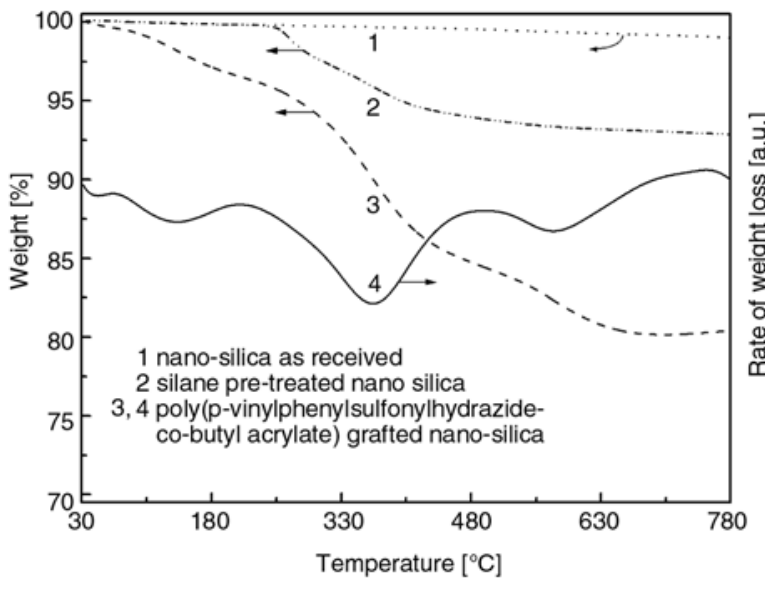

Figure 3. TGA thermograms of the related materials. For the grafted nano-silica, the ungrafted polymer had been removed by solvent extraction before the TGA measurement

result of poly(p-vinylphenylsulfonylhydrazide) grafted ones and ranks between the gasification temperatures of p-vinylphenylsulfonylhydrazide $\left(120^{\circ} \mathrm{C}\right)$ and poly(p-vinylphenylsulfonylhydrazide) $\left(169^{\circ} \mathrm{C}\right)$ as reported in our previous work [5]. It must originate from gasification of the sulfonyl hydrazide groups. Clearly, copolymerization of pvinylphenylsulfonylhydrazide with butyl acrylate does not change blowing ability of the former. As a result, poly(p-vinylphenylsulfonylhydrazide-cobutyl acrylate) grafted silica nanoparticles should be able to arouse in-situ bubble-stretching when the particles are blended with PP melt.

To demonstrate the effect of the grafted nanoparticles, TEM photos of the PP composites were compared. As shown in Figure 4, the grafted nanoparticles seems to be much smaller than the untreated ones in the polymer matrix. Distribution of the treated nano-silica is rather homogeneous (Figure 4b). Evidently, the local rapid expansion of

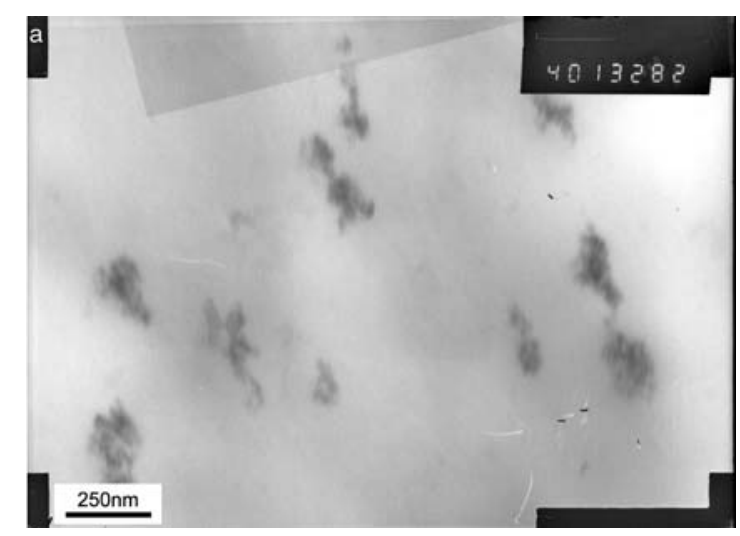

Figure 4. TEM images of ultrathin sections of (a) untreated nano- $\mathrm{SiO}_{2} / \mathrm{PP}$, (b) poly(p-vinylphenylsulfonylhydrazide-co-

butyl acrylate) grafted nano- $\mathrm{SiO}_{2} / \mathrm{PP}$ composites

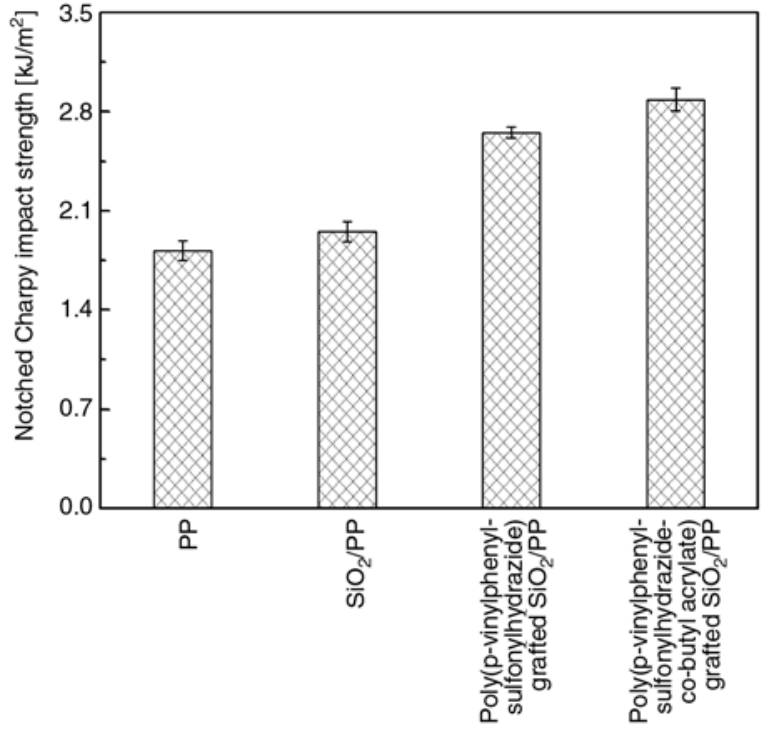

Figure 5. Notched Charpy impact strength of PP and its composites. Content of nano-silica in all the composites: $1.43 \mathrm{vol} \%$

the matrix polymer nearby the nanoparticles caused by gasification of the sulfonyl hydrazide groups on the grafted polymer must have taken effect. It forces $\mathrm{SiO}_{2}$ nanoparticle agglomerates to be deagglomerated in the course of melt compounding.

With respect to mechanical properties of the nanocomposites, Figure 5 shows the results of notched Charpy impact strength. It is seen that the contribution of the untreated fillers to the improvement of toughness under high speed deformation and to the reduction of notch sensitivity is nearly negligible as compared with the grafted ones. This should be attributed to the worse dispersion of the untreated nanoparticles in PP matrix. Besides, the loose clusters of the nanoparticles might act as weak sites of the composites. For poly(p-vinylphenylsulfonylhydrazide-co-butyl acrylate) grafted $\mathrm{SiO}_{2} / \mathrm{PP}$ composite, its notched impact strength is higher than

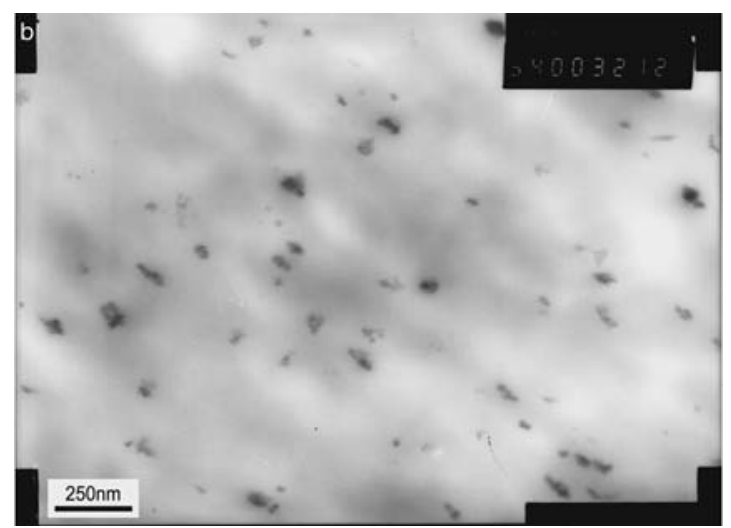


that of poly(p-vinylphenylsulfonylhydrazide) grafted $\mathrm{SiO}_{2} / \mathrm{PP}$ system. According to Rong et al. [7], the grafted polymer, especially the one with higher molecular mobility, might play the role of a bumper interlayer around the fillers, while absorbing impact energy and preventing initiation of cracks. The data in Figure 5 coincide with the above conclusion. It means that even dispersion of the nanoparticles driven by in-situ bubble stretching does not guarantee the optimal toughening effect. An increase in flexibility of the grafted polymer chains facilitates a further improvement of nanocomposites' ductility.

Tensile properties of PP composites are illustrated in Figure 6. In comparison with neat PP, all the composites exhibit higher tensile strength. A careful survey indicates that the increase in strength of untreated nano-silica/PP composite is marginal while that of grafted nano-silica/PP composites are more prominent. The chain entanglement/ interdiffusion at the interface between the grafted polymer and PP matrix should account for the strengthening effect, due to the enhanced stress transfer efficiency [8]. This is different from the situation of microparticulates/polymer composites, which is characterized by a drastic decrease in tensile strength with the incorporation of the fillers [9].

It is interesting to see that tensile strengths of poly(pvinylphenylsulfonylhydrazide) grafted nano-silica/PP and poly(p-vinylphenylsulfonylhydrazideco-butyl acrylate) grafted nano-silica/PP composites are almost the same regardless of species of the

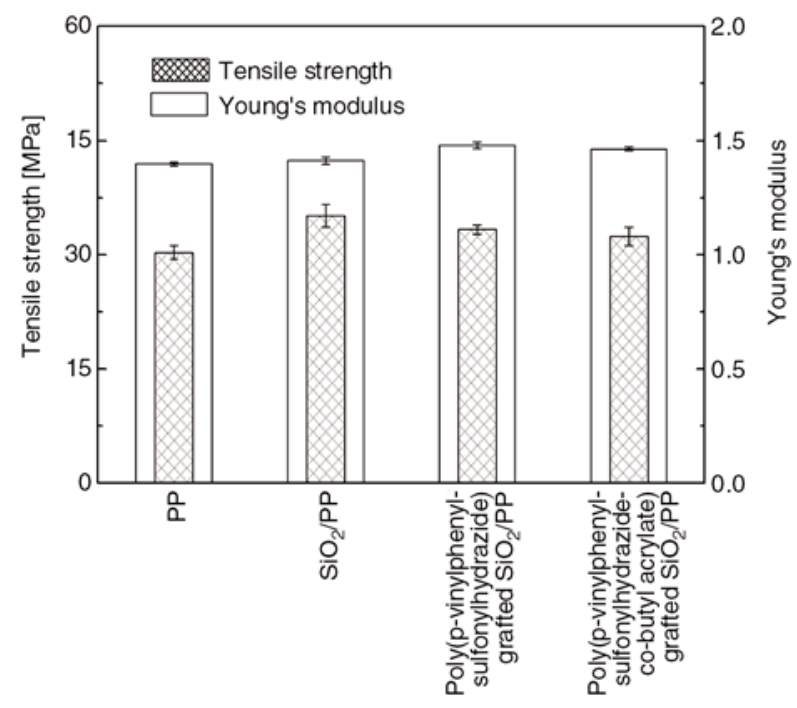

Figure 6. Tensile strength and Young's modulus of PP and its composites. Content of nano-silica in all the composites: $1.43 \mathrm{vol} \%$ grafted polymer. Obviously, the difference in rigidity of the interphase in the two nanocomposites is not extremely great, which ensures similar stress transfer behavior under static tensile condition. The analysis receives support from Young's modulus of the composites, as both grafted nano-silica/PP composites possess nearly the same stiffness (Figure 6). It suggests that the mechanical responses of the composites containing two types of grafted polymer resemble each other within Hooke's limit. On the other hand, because Young's modulus of particulate composites is highly related to the fillermatrix interfacial interaction [10], appearance of grafted polymer on the nanoparticles has to shield the matrix from the particles and leads to insufficient impartation of the fillers' rigidity to PP. Therefore, the untreated $\mathrm{SiO}_{2} / \mathrm{PP}$ composite has the highest Young's modulus.

\section{Conclusions}

The above investigation reveals the importance of both deagglomeration of nanoparticles and interfacial design in making polymer nanocomposites with improved properties. Taking the advantage of graft polymerization, the specific structural requirements can be satisfied in one step. The key issue lies in the introduction of polymeric foaming agent containing soft segments (i.e. poly(p-vinylphenylsulfonylhydrazide-co-butyl acrylate)) onto the surface of nano- $\mathrm{SiO}_{2}$. In the case of melt blending with $\mathrm{PP}$, the side sulfonyl hydrazide groups on the grafted copolymer are able to be gasified like foaming agent to induce localized bubble-stretching effect that pulls apart nanoparticles agglomerates. Meanwhile, the skeleton of the grafted copolymer would get entangled with the matrix polymer forming strong interfacial interaction, and the poly(butyl acrylate) units in the grafted copolymer help to raise ductility of the interlayer. Compared to the composite containing rigid macromolecular foaming agent grafted nano- $\mathrm{SiO}_{2}$ (i.e., poly(p-vinylphenylsulfonylhydrazide) grafted nano- $\mathrm{SiO}_{2} / \mathrm{PP}$ ), the composite fabricated in this work (i.e., poly(pvinylphenylsulfonylhydrazide-co-butyl acrylate) grafted nano- $\mathrm{SiO}_{2} / \mathrm{PP}$ ) shows much greater increment in notched impact strength without expense of lowing tensile performance. In consideration of structure adjustability of copolymers attached to nanoparticles, the present work has opened up a 
simple way to manufacture polymer nanocomposites with tailorable properties.

\section{Acknowledgements}

The authors are grateful for the support of the National Natural Science Foundation of China (Grant: 50473019), the Natural Science Foundation of Guangdong, China (Grants: 2004A10702001, 2005A10702001 and 5003267), the Science and Technology Department of Guangzhou, China (Grant: 2004Z3-D2061), and the Guangdong Economic \& Trade Commission, China (Grant: 20040112).

\section{References}

[1] Zhang M. Q., Rong M. Z., Friedrich K.: Processing and properties of nonlayered nanoparticle reinforced thermoplastic composites. in: 'Handbook of organicinorganic hybrid materials and nanocomposites' (ed.: Nalwa H. S.) American Science Publishers, Los Angeles, Vol 2, 113-150 (2003).

[2] Leuteritz A., Pospiech D., Kretzschmar B., Willeke M., Jehnichen D., Jentzsch U., Grundke K., Janke A.: Progress in polypropylene nanocomposites development. Advanced Engineering Materials, 5, 678-681 (2003).

[3] Rong M. Z., Zhang M. Q., Ruan W. H.: Surface modification of nanoscale fillers for improving properties of polymer nanocomposites: a review. Materials Science and Technology, 22, 787-796 (2006).
[4] Meng Q. Y., Wu D. M.: A study of bubble inflation in polymers and its applications. Physics Letters A., 327, 61-66 (2004).

[5] Cai L. F., Huang X. B., Rong M. Z., Ruan W. H., Zhang M. Q.: Effect of grafted polymeric foaming agent on the structure and properties of nano-silica/ polypropylene composites. Polymer, 47, 7043-7050 (2006).

[6] Keszei S., Matko S., Bertalan G., Anna P., Marosi G., Toth A.: Progress in interface modifications: from compatibilization to adaptive and smart interphases. European Polymer Journal, 41, 697-705 (2005).

[7] Rong M. Z., Zhang M. Q., Pan S. L., Friedrich K.: Interfacial effects in polypropylene-silica nanocomposites. Journal of Applied Polymer Science, 92, 1771-1781 (2004).

[8] Wu C. L., Zhang M. Q., Rong M. Z., Friedrich K.: Tensile performance improvement of low nanoparticles filled-polypropylene composites. Composites Science and Technology, 62, 1327-1340 (2002).

[9] Nicolais L., Narkis M.: Stress-strain behavior of styrene-acrylonitrile/glass bead composites in the glassy region. Polymer Engineering and Science, 11, 194-199 (1971).

[10] Walter R., Friedrich K., Privalko V., Savadori A.: On modulus and fracture toughness of rigid particulate filled high density polyethylene. Journal of Adhesion, 64, 87-109 (1997). 